Internat. J. Math. \& Math. Sci.

Vol. 23, No. 11 (2000) 729-739

S0161171200003288

(C) Hindawi Publishing Corp.

\title{
QUANTIFYING COMPLETION
}

\section{ROBERT LOWEN and BART WINDELS}

\author{
(Received 23 April 1999)
}

\begin{abstract}
Approach uniformities were introduced in Lowen and Windels (1998) as the canonical generalization of both metric spaces and uniform spaces. This text presents in this new context of "quantitative" uniform spaces, a reflective completion theory which generalizes the well-known completions of metric and uniform spaces. This completion behaves nicely with respect to initial structures and hyperspaces. Also, continuous extensions of pseudo-metrics on uniform spaces and (real) compactification of approach spaces can be interpreted in terms of this completion.
\end{abstract}

Keywords and phrases. Completion, uniform space, approach space, approach uniformity, compactification, realcompactification.

2000 Mathematics Subject Classification. Primary 54E15, 18B30, 54B20.

1. Approach uniformities. The motivation for the introduction of approach uniformities (which is the subject of Lowen and Windels [6]) is twofold. On the one hand, the category AP of approach spaces (see Lowen [3]) is not fit to handle uniform concepts. Different results concerning completeness in the category AP imply that the theory is essentially local. For instance, the space of continuous functions between metric spaces, equipped with the pointwise distance, turns out to be complete. On the other hand, AP seems not to be the right context for the quantification of uniform properties, such as completeness and total boundedness, whereas for topological concepts everything works out quite well in AP. Therefore, it was natural to seek a new category that combines the quantitative aspects of AP (or pMET) and qualitative uniform concepts. To that end the concept of approach uniformities was introduced in [6].

For the reader's convenience we briefly recall the main definitions and results. An approach uniform space $(X, \Gamma)$ is a set $X$ together with an ideal $\Gamma$ of functions from $X \times X$ into $[0, \infty]$, satisfying the following conditions:

(AU1) $\forall \gamma \in \Gamma, \forall x \in X: \gamma(x, x)=0$.

(AU2) $\forall \xi \in[0, \infty]^{X \times X}:\left(\forall \varepsilon>0, \forall N<\infty: \exists \gamma_{\varepsilon}^{N} \in \Gamma\right.$ s.t. $\left.\xi \wedge N \leq \gamma_{\varepsilon}^{N}+\varepsilon\right) \Rightarrow \xi \in \Gamma$.

(AU3) $\forall \gamma \in \Gamma, \forall N<\infty, \exists \gamma^{N} \in \Gamma$ s.t. $\forall x, y, z \in X: \gamma(x, z) \wedge N \leq \gamma^{N}(x, y)+\gamma^{N}(y, z)$.

(AU4) $\forall \gamma \in \Gamma: \gamma^{s} \in \Gamma$.

Equivalently, an approach uniform space can be described by means of a uniform tower, i.e., a family of filters $\left(\mathfrak{U}_{\varepsilon}\right)_{\varepsilon \in \mathbb{R}^{+}}$on $X \times X$, such that

(UT1) $\forall \varepsilon \in \mathbb{R}^{+}, \forall U \in \mathfrak{U}_{\varepsilon}: \Delta_{X} \subset U$.

(UT2) $\forall \varepsilon \in \mathbb{R}^{+}, \forall U \in \mathfrak{U}_{\varepsilon}: U^{-1} \in \mathfrak{U}_{\varepsilon}$.

(UT3) $\forall \varepsilon, \varepsilon^{\prime} \in \mathbb{R}^{+}: \mathfrak{U}_{\varepsilon} \circ \mathfrak{U}_{\varepsilon^{\prime}} \supset \mathfrak{U}_{\varepsilon+\varepsilon^{\prime}}$.

(UT4) $\forall \varepsilon \in \mathbb{R}^{+}: \mathfrak{U}_{\varepsilon}=\bigcup_{\alpha>\varepsilon} \mathfrak{U}_{\alpha}$.

or equivalently, a family $\left(\mathfrak{U}_{\varepsilon}\right)_{\varepsilon \in \mathbb{R}^{+}}$of semi-uniformities, satisfying (UT3) and (UT4). 
For example, if $d$ is a pseudo-metric, then the collection $\Gamma(d):=\{\gamma \mid \gamma \leq d\}$ is an approach uniformity. It is referred to as the metric approach uniformity induced by $d$.

If $\mathfrak{U}$ is a uniformity, then the trivial tower $(\mathfrak{U})_{\varepsilon}(\mathfrak{U}$ on every level $\varepsilon)$, is a uniform tower, defining an approach uniformity $\Gamma(\mathfrak{U})$, which is referred to as the uniform approach uniformity induced by $\mathfrak{U}$. If $(X, \Gamma)$ and $(Y, \Psi)$ are approach uniform spaces, then a function $f:(X, \Gamma) \rightarrow(Y, \Psi)$ is called a uniform contraction if and only if $\forall \psi \in$ $\Psi: \psi \circ(f \times f) \in \Gamma$.

The category AUnif of approach uniform spaces and uniform contractions is a topological category. It contains Unif both reflectively and coreflectively and pMET coreflectively.

Approach uniformities establish a context for quantifying uniform concepts. In [5], a measure of total boundedness (and precompactness), of completeness and of uniform connectedness is presented.

For every approach uniformity $(X, \Gamma)$ and for any $x \in X$ we can consider the set

$$
\mathcal{H}(x):=\{\gamma(x, \cdot) \mid \gamma \in \Gamma\} \subset[0, \infty]^{X} .
$$

The family $(\mathcal{A}(x))_{x \in X}$ defines an ordinary approach structure on $X$, which we shall call the underlying approach structure of $\Gamma$. This procedure yields a forgetful functor A: AUnif $\rightarrow$ AP.

Also recall that in any approach space $\left(X,(\mathcal{A}(x))_{x \in X}\right)$ and for any filter $\mathfrak{f}$ on $X$ and any $x \in X$, we denote

$$
\lambda \mathfrak{f}(x):=\sup _{\varphi \in \mathfrak{A}(x)} \inf _{F \in \mathfrak{f}} \sup _{y \in F} \varphi(y)
$$

2. Cauchy filters. Let $X$ be a set, and let $U \subset X \times X$. Then we denote

$$
\theta_{U}: X \times X \longrightarrow[0, \infty]: \begin{cases}(x, y) \longmapsto 0 & \text { if }(x, y) \in U, \\ (x, y) \longmapsto 1 & \text { if }(x, y) \notin U .\end{cases}
$$

Proposition 2.1. Let $(X, \Gamma)$ be an approach uniform space, and let $\mathfrak{f}$ be a filter on $X$. Then the following are equivalent.

(1) $\mathfrak{f}$ is Cauchy with respect to the uniform coreflection.

(2) $\forall \gamma \in \Gamma, \forall \varepsilon>0, \exists F \in \mathcal{f}: \theta_{F \times F}+\varepsilon>\gamma$.

Proof. $\mathfrak{f}$ is Cauchy with respect to the uniform coreflection $\mathfrak{U}_{0}$ if and only if

$$
\begin{aligned}
\forall U \in \mathcal{U}_{0}, & \exists F \in \mathfrak{f}: F \times F \subset U \\
& \Longleftrightarrow \forall \gamma \in \Gamma, \forall \varepsilon>0, \exists F \in \mathfrak{f}: F \times F \subset\{\gamma<\varepsilon\} . \\
& \Longleftrightarrow \forall \gamma \in \Gamma, \forall \varepsilon>0, \exists F \in \mathfrak{f}: \theta_{F \times F}+\varepsilon>\gamma .
\end{aligned}
$$

A filter on $(X, \Gamma)$ is called $(\Gamma-)$ Cauchy if and only if it satisfies the conditions of the previous proposition.

DEFinition 2.2. Two filters $\mathfrak{f}$ and $\mathfrak{g}$ on $(X, \Gamma)$ are called equivalent if and only if $\mathfrak{f} \cap \mathfrak{g}$ is Cauchy. 
In Lowen [3] Cauchy filters in approach spaces are introduced: a filter $\mathfrak{f}$ on $X$ is called Cauchy with respect to the approach space $\left(X,(\mathcal{H}(x))_{x}\right)$ if and only if $\inf _{x \in X} \lambda \mathfrak{f}(x)=0$. This definition yields a categorically nice completion theory in AP (see [3]).

We have the following relationship between AP-Cauchy filters and AUnif-Cauchy filters.

Proposition 2.3. Let $(X, \Gamma)$ be an approach uniform space, and let $(X, A(\Gamma))$ denote its underlying approach space. For any filter $\mathfrak{f}$ on $X$, we have

$$
\mathfrak{f} \text { is } A(\Gamma) \text {-Cauchy } \Longrightarrow \mathfrak{f} \text { is } \Gamma \text {-Cauchy. }
$$

Proof. $\mathfrak{f}$ is $A(\Gamma)$-Cauchy if and only if $\inf _{x \in X} \lambda \mathfrak{f}(x)=0$, i.e., if and only if

$$
\forall \varepsilon>0, \exists x \in X, \forall \gamma \in \Gamma, \exists F \in \mathfrak{f}, \forall y \in F: \gamma(x, y)<\varepsilon .
$$

Let $\gamma \in \Gamma$ and $\varepsilon>0$. Take $\gamma^{N} \in \Gamma, \gamma^{N}$ symmetric, such that $\forall x, y, z \in X: \gamma(x, z) \wedge N \leq$ $\gamma^{N}(x, y)+\gamma^{N}(y, z)$ for some $N>\varepsilon$, and take $x_{0} \in X$ and $F \in \mathfrak{f}$ such that $\forall y \in F$ : $\gamma^{N}\left(x_{0}, y\right)<\varepsilon / 2$. Clearly, we have $\forall y, z \in F$ :

$$
\gamma(y, z) \wedge N \leq \gamma^{N}\left(y, x_{0}\right)+\gamma^{N}\left(x_{0}, z\right) \leq \varepsilon .
$$

Consequently,

$$
\forall \gamma \in \Gamma, \forall \varepsilon>0, \exists F \in \mathcal{f}, \forall x, y \in F: \gamma(x, y) \leq \varepsilon,
$$

which means that $f$ is $\Gamma$-Cauchy.

Proposition 2.4. Let $(X, \Gamma)$ be an approach uniformity, and let $\mathfrak{f}$ be a Cauchy filter on $X$. Then $\mathfrak{z}:=\left\{\left\{y \mid \inf _{x \in F} \gamma(x, y)<\alpha\right\} \mid \gamma \in \Gamma, \alpha>0, F \in \mathfrak{f}\right\}$ is a filterbasis for $a$ Cauchy filter $2 \mathrm{~W}$, coarser than $\mathfrak{f}$. Moreover, $2 \mathrm{~W}$ is the coarsest Cauchy filter with that property. Consequently, $2 \mathrm{~b}$ and $\mathfrak{f}$ are equivalent.

Proof. Let $\mathfrak{U}_{0}$ denote the uniform coreflection of $\Gamma$. The proof becomes folklore, if one observes that

$$
\mathfrak{b}=\left\{U(F) \mid U \in \mathfrak{U}_{0}, F \in \mathfrak{f}\right\} .
$$

DEFINITION 2.5. An approach uniformity $(X, \Gamma)$ is called complete if and only if every $\Gamma$-Cauchy filter converges (with respect to the underlying topology of the uniform coreflection of $\Gamma$ ).

Thus an approach uniformity is called complete if and only if its uniform coreflection is complete.

By Proposition 2.3, we know that if $(X, \Gamma)$ is AUnif-complete, then $(X, A(\Gamma))$ is APcomplete. The converse need not be true.

EXAMPLE 2.6. Let $(E,\|\cdot\|)$ be a non-reflexive Banach space. If $E$ is equipped with the initial AP-structure $\delta$ for the source

$$
(f: E \longrightarrow \mathbb{R})_{f \in E^{\star},\|f\|_{E^{\star}} \leq 1},
$$

(where $\mathbb{R}$ is equipped with the usual distance), then $E$ is complete in AP. 
However, if $E$ is equipped with the initial AUnif-structure $\Gamma$ for the source

$$
(f: E \longrightarrow \mathbb{R})_{f \in E^{\star},\|f\|_{E^{\star} \leq 1}},
$$

(where $\mathbb{R}$ is equipped with the usual approach uniformity), then $E$ is not complete in AUnif.

Indeed, we know that $\forall_{x} \in E, \forall A \subset E$

$$
\delta(x, A)=\sup _{\left.F \in 2^{\left(E^{\star}\right.}\right)} \inf _{y \in A} \sup _{f \in F}|f(x)-f(y)|,
$$

and therefore the metric coreflection $d_{\delta}$ is given by $\forall x, y \in E$

$$
d_{\delta}(x, y)=\sup _{F \in 2^{\left(E^{\star}\right)}} \sup _{f \in F}|f(x)-f(y)|=\|x-y\| .
$$

Since $E$ is Banach, $(E, \delta)$ is complete.

By [6, Proposition 2.16] we know that the uniform coreflection $\mathfrak{U}_{0}$ of $\Gamma$ is initial for the Unif-source

$$
(f: E \rightarrow \mathbb{R})_{f \in E^{\star},\|f\|_{E^{\star} \leq 1}},
$$

(where $\mathbb{R}$ is equipped with the usual uniformity). Let $B:=\{x \in E \mid\|x\| \leq 1\}$. Then $f(B) \subset[-1,1]$, which is totally bounded. Thus $B$ is totally bounded. However, $B$ is not compact, since $E$ is non-reflexive, hence $B$ is not complete. Therefore $(E, \Gamma)$ is not complete.

3. Completion. By Proposition 2.4, it makes sense to define $\hat{X}$ to be the set of all minimal Cauchy filters on $X$.

Proposition 3.1. Let $(X, \Gamma)$ be an approach uniformity. If $\gamma \in \Gamma$, define $\forall 2 \mathfrak{W}, 2 \tau \in \hat{X}$

$$
\hat{\gamma}(2 \mathfrak{W}, 2 \mathfrak{2}):=\inf _{F \in \mathfrak{2} \cap 2 \mathfrak{2}} \sup _{x, y \in F} \gamma(x, y) \text {. }
$$

Then $\hat{\Gamma}:=\langle\{\hat{\gamma} \mid \gamma \in \Gamma, \gamma$ symmetric $\}\rangle$ is an approach uniformity on $\hat{X}$.

Proof. The fact that $\hat{\Gamma}$ is an ideal follows from the observation that $\forall \gamma_{1}, \gamma_{2} \in \Gamma$ : $\hat{\gamma}_{1} \vee \hat{\gamma}_{2} \leq \widehat{\gamma_{1} \vee \gamma_{2}}$.

(AU1) Let $\gamma \in \Gamma$ and $2 \mathfrak{W} \in \hat{X}$. Since $2 \mathfrak{W}$ is Cauchy, we have that $\forall \varepsilon>0, \exists F \in 2 \mathfrak{W}$ :

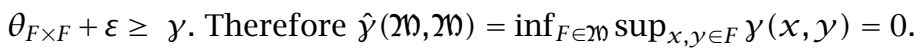

(AU2) This is obvious.

(AU3) Let $\gamma \in \Gamma$ and $N<\infty$. Then there is some $\gamma^{N} \in \Gamma$ such that $\forall x, y, z \in X$ : $\gamma(x, z) \wedge N \leq \gamma^{N}(x, y)+\gamma^{N}(y, z)$. We shall prove that $\forall 2 \mathfrak{W}, 2 \mathfrak{2}, \mathfrak{f} \in \hat{X}: \hat{\gamma}(\mathfrak{W}, 2 \mathfrak{l}) \wedge N \leq$ $\hat{\gamma}^{N}(\mathfrak{2} \mathfrak{W}, \mathfrak{f})+\hat{\gamma}^{N}(\mathfrak{f}, 2 \mathfrak{2})$. Let $F_{1} \in \mathfrak{f} \cap 2 \mathfrak{W}$ and $F_{2} \in \mathfrak{f} \cap 2 \mathfrak{2}$. Then $F:=F_{1} \cup F_{2} \in \mathfrak{W} \cap 2 \pi$. Since $F_{1} \cap F_{2} \neq \varnothing$, we have that

$$
\sup _{x, y \in F} \gamma(x, y) \wedge N \leq \sup _{x, z \in F_{1}} \gamma^{N}(x, z)+\sup _{z, y \in F_{2}} \gamma^{N}(z, y),
$$

and therefore

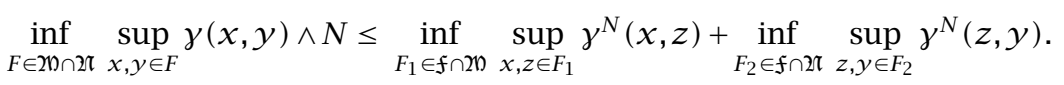

(AU4) This follows from the observation that $\forall \gamma \in \Gamma: \hat{\gamma}^{s}=\hat{\gamma}^{s}$. 
The approach uniformity $(\hat{X}, \hat{\Gamma})$ in the previous proposition is called the completion of $(X, \Gamma)$. We have the following obvious relationship between the completion of the uniform coreflection, and the uniform coreflection of the completion.

Proposition 3.2. If $\left(\mathfrak{U}_{\varepsilon}\right)_{\varepsilon}$ and $\left(\hat{\mathfrak{U}}_{\varepsilon}\right)_{\varepsilon}$ are the towers of $(X, \Gamma)$ and $(\hat{X}, \hat{\Gamma})$ respectively, then $(\hat{\mathfrak{U}})_{0}$ coincides with the uniform completion $\hat{\mathfrak{U}}_{0}$ of $\mathfrak{U}_{0}$.

Proof. We see that

$$
\begin{aligned}
& (\hat{\mathfrak{U}})_{0}=\langle\{\{\hat{\gamma}<\alpha\} \mid \hat{\gamma} \in \hat{\Gamma}, \alpha>0\}\rangle \\
& =\langle\{(2 \mathcal{W}, 2 \mathcal{T}) \mid \exists F \in \mathfrak{W} \cap 2 \mathcal{W}, \forall a, b \in F: \gamma(a, b)<\alpha\}\rangle \\
& =\langle\{(2 \mathfrak{W}, 2 \mathfrak{l}) \mid \exists F \in 2 \mathfrak{W} \cap 2 \mathfrak{\tau}: F \times F \subset\{\gamma<\alpha\}\}\rangle \\
& =\hat{\mathfrak{U}}_{0} \text {. }
\end{aligned}
$$

From now on we can write $\hat{\mathfrak{U}}_{0}$ without ambiguity.

Before we move on, we check that completion behaves nicely with respect to bases. An ideal basis $\mathbb{D}$ in $[0, \infty]^{X \times X}$ is called a basis for $\Gamma$, if $\mathbb{D}$ satisfies (AU1), (AU2), and (AU4) and

$$
\Gamma=\langle\mathbb{D}\rangle:=\left\{\xi \in[0, \infty]^{X \times X} \mid \forall \varepsilon>0, \forall N<\infty: \exists \gamma_{\varepsilon}^{N} \in \mathbb{D}: \xi \wedge N \leq \gamma_{\varepsilon}^{N}+\varepsilon\right\} .
$$

Proposition 3.3. If $\langle\mathcal{D}\rangle=\Gamma$, then $\langle\{\hat{d} \mid d \in \mathbb{D}\}\rangle=\hat{\Gamma}$.

Proof. This is a direct consequence of the fact that $\forall \gamma, \xi \in[0, \infty]^{X \times X}, \forall \varepsilon>0$, $\forall N<\infty$ :

$$
\xi \wedge N \leq \gamma+\varepsilon \Rightarrow \hat{\xi} \wedge N \leq \hat{\gamma}+\varepsilon
$$

If $x \in X$, then let $\mathfrak{v}_{x}$ denote the neighbourhoodfilter of $x$ with respect to the underlying topology of the uniform coreflection.

Proposition 3.4. Let $(X, \Gamma)$ be an approach uniformity. Then

$$
i:(X, \Gamma) \longrightarrow(\hat{X}, \hat{\Gamma}) \quad x \longmapsto \mathfrak{V}_{x}
$$

is initial, and $i(X)$ is dense in $\hat{X}$. Moreover, if $(X, \Gamma)$ is Hausdorff, then $i$ is an embedding.

Proof. It is well-known (cf. [10]) that $\forall x \in X: \mathfrak{V}_{x}$ is a minimal Cauchy filter, and therefore $i$ is well-defined; and also, that $i(X)$ is dense in $\hat{X}$.

In order to show that $\Gamma$ is the initial structure, first notice that if $\gamma \in \Gamma$, then $\forall x, y \in X$ :

$$
\hat{\gamma} \circ(i \times i)(x, y)=\hat{\gamma}\left(\mathfrak{V}_{x}, \mathfrak{D}_{y}\right)=\inf _{F \in \mathfrak{V}_{x} \cap \mathfrak{v}_{y}} \sup _{a, b \in F} \gamma(a, b) .
$$

Let $\gamma \in \Gamma$ be bounded. Let $\tilde{\gamma} \in \Gamma$ be such that $\forall x, y, z, u \in X: \gamma(x, u) \leq \tilde{\gamma}(x, y)+$ $\tilde{\gamma}(y, z)+\tilde{\gamma}(z, u)$. We shall prove that

(1) $\gamma \leq \hat{\gamma} \circ(i \times i)$.

(2) $\hat{\gamma} \circ(i \times i) \leq \tilde{\gamma}$.

Proof of (1). Let $x, y \in X$. Suppose $\hat{\gamma} \circ(i \times i)(x, y)<M \in \mathbb{R}^{+}$, then there is some $F \in \mathfrak{V}_{x} \cap \mathfrak{V}_{y}$ such that $\forall a, b \in F: \gamma(a, b)<M$. Since $\{x, y\} \subset F$, this means that $\gamma(x, y)<M$. 
Proof OF (2). Let $x, y \in X$ and $\varepsilon>0$. Put $F:=\{z \in X \mid \tilde{\gamma}(x, z)<\varepsilon / 2$ or $\tilde{\gamma}(y, z)<$ $\varepsilon / 2\} \in \mathfrak{D}_{x} \cap \mathfrak{D}_{y}$. Let $a, b \in F$.

- If $\tilde{\gamma}(x, a)<\varepsilon / 2$ and $\tilde{\gamma}(x, b)<\varepsilon / 2$, then $\gamma(a, b) \leq \tilde{\gamma}(a, x)+\tilde{\gamma}(x, b)<\varepsilon$.

- If $\tilde{\gamma}(y, a)<\varepsilon / 2$ and $\tilde{\gamma}(y, b)<\varepsilon / 2$, then also $\gamma(a, b)<\varepsilon$.

- If $\tilde{\gamma}(x, a)<\varepsilon / 2$ and $\tilde{\gamma}(y, b)<\varepsilon / 2$, then $\gamma(a, b) \leq \tilde{\gamma}(a, x)+\tilde{\gamma}(x, y)+\tilde{\gamma}(y, b) \leq$ $\tilde{\gamma}(x, y)+\varepsilon$.

- If $\tilde{\gamma}(x, b)<\varepsilon / 2$ and $\tilde{\gamma}(y, a)<\varepsilon / 2$, then $\gamma(a, b) \leq \tilde{\gamma}(x, y)+\varepsilon$ too.

In any case $\sup _{a, b \in F} \gamma(a, b) \leq \tilde{\gamma}(x, y)+\varepsilon$, and thus $\hat{\gamma} \circ(i \times i) \leq \tilde{\gamma}+\varepsilon$, which by arbitrariness of $\varepsilon$ proves the claim.

If $(X, \Gamma)$ is Hausdorff, then for all $x \neq y: \mathfrak{v}_{x} \neq \mathfrak{v}_{y}$ and therefore $i$ is an embedding.

Now we have to prove that a completion really is complete.

Proposition 3.5. Let $(X, \Gamma)$ be an approach uniformity. Then $(\hat{X}, \hat{\Gamma})$ is a complete approach uniform space.

Proof. This is a consequence of Proposition 3.2.

Proposition 3.6. Let $(Y, \Psi)$ be a Hausdorff complete approach uniformity. If $f$ : $(X, \Gamma) \rightarrow(Y, \Psi)$ is a uniform contraction, then there is a unique uniform contraction $\hat{f}:(\hat{X}, \hat{\Gamma}) \rightarrow(Y, \Psi)$ such that $\hat{f} \circ i=f$.

PROoF. If $\left(\mathfrak{U}_{\varepsilon}\right)_{\varepsilon}$ and $\left(\mathfrak{V}_{\varepsilon}\right)_{\varepsilon}$ are the towers of $\Gamma$ and $\Psi$ respectively, then $f:\left(X, \mathfrak{U}_{0}\right) \rightarrow$ $\left(X, \mathfrak{V}_{0}\right)$ is uniformly continuous and $\left(Y, \mathfrak{V}_{0}\right)$ is a Hausdorff complete uniform space. It is well-known that there is a unique $\hat{f}:\left(\hat{X}, \hat{\mathfrak{U}}_{0}\right) \rightarrow\left(Y, \mathfrak{V}_{0}\right)$ uniformly continuous such that $\hat{f} \circ i=f$. In fact we know that for all $2 \mathfrak{W} \in \hat{X}: \hat{f}(\mathfrak{W})=\lim f\left(\mathfrak{V}_{2 \mathfrak{D}} \mid X\right)$. We shall prove that $\hat{f}$ is a uniform contraction. To that end, let $\psi \in \Psi$; we shall show that $\psi \circ(\hat{f} \times \hat{f}) \in \hat{\Gamma}$.

Recall that if $2 \mathrm{~b}$ is a Cauchy-filter, then

$$
\forall \gamma \in \Gamma, \forall \alpha>0, \forall F \in M, \exists x \in F, \exists M \in \mathfrak{W} \cap \mathfrak{D}_{x}, \forall a, b \in M: \gamma(a, b)<\alpha .
$$

Let $2 \mathfrak{W}, 2 \mathfrak{2} \in \hat{X}$ and let $\varepsilon>0$. We have that $f\left(\mathfrak{V}_{2 \mathfrak{m}} \mid X\right) \rightarrow \hat{f}(\mathfrak{2} \mathfrak{W})$, i.e., $f\left(\mathfrak{V}_{2 \mathfrak{m}} \mid X\right) \supset$ $2 \pi(\hat{f}(2 \mathfrak{W}))$. Since

$$
\begin{aligned}
& f\left(\mathfrak{V}_{2 \mathfrak{b}} \mid X\right)=\left\langle\left\{\left\{f(x) \mid \hat{\gamma}\left(\mathfrak{2} \mathfrak{W}, \mathfrak{V}_{x}\right)<\alpha\right\} \mid \gamma \in \Gamma, \alpha>0\right\}\right\rangle \\
& \mathfrak{I}(\hat{f}(\mathfrak{W}))=\langle\{\{y \mid \psi(\hat{f}(\mathfrak{W}, y))<\beta\} \mid \psi \in \Psi, \beta>0\}\rangle,
\end{aligned}
$$

we find that

$\forall \beta>0, \forall \psi \in \Psi, \exists \alpha_{1}>0, \exists \gamma_{1} \in \Gamma, \forall x \in X: \gamma_{1}\left(\mathfrak{W}, \mathfrak{v}_{x}\right)<\alpha_{1} \Longrightarrow \psi(\hat{f}(\mathfrak{W}), f(x))<\beta$

Analogously we have that

$$
\forall \beta>0, \forall \psi \in \Psi, \exists \alpha_{2}>0, \exists \gamma_{2} \in \Gamma, \forall x \in X: \gamma_{2}\left(\mathfrak{2} \mathfrak{T}, \mathfrak{D}_{x}\right)<\alpha_{2} \Longrightarrow \psi(\hat{f}(2 \mathfrak{l}), f(x))<\beta .
$$


Let $\tilde{\psi} \in \Psi$ such that $\forall x, y, z, u \in Y: \psi(x, u) \leq \tilde{\psi}(x, y)+\tilde{\psi}(y, z)+\tilde{\psi}(z, u)$. Then we obtain that there exist $\alpha>0$ and $\gamma \in \Gamma$ such that for all $x \in X$ :

$$
\hat{\gamma}\left(2 \mathfrak{W}, \mathfrak{b}_{x}\right)<\alpha \Longrightarrow \tilde{\psi}(\hat{f}(\mathfrak{2} \mathfrak{W}), f(x))<\frac{\varepsilon}{2}, \quad \hat{\gamma}\left(2 \mathfrak{i}, \mathfrak{b}_{x}\right)<\alpha \Longrightarrow \tilde{\psi}(\hat{f}(2 \mathfrak{l}), f(x))<\frac{\varepsilon}{2} .
$$

From (3.9) we deduce that $\forall F \in 2 \mathfrak{W} \cap 2 \mathfrak{l}$ :

$$
\exists x_{1} \in F: \hat{\gamma}\left(2 \mathfrak{W}, \mathfrak{v}_{x_{1}}\right)<\alpha, \quad \exists x_{2} \in F: \hat{\gamma}\left(2 \mathfrak{T}, \mathfrak{v}_{x_{2}}\right)<\alpha,
$$

and thus

$$
\exists x_{1} \in F: \tilde{\psi}\left(\hat{f}(2 \mathcal{D}), f\left(x_{1}\right)\right)<\frac{\varepsilon}{2}, \quad \exists x_{2} \in F: \tilde{\psi}\left(\hat{f}(2 \mathfrak{l}), f\left(x_{2}\right)\right)<\frac{\varepsilon}{2} .
$$

Then

$$
\begin{aligned}
\psi(\hat{f}(2 \mathfrak{W}), \hat{f}(2 \mathfrak{l})) & \leq \tilde{\psi}\left(\hat{f}(\mathfrak{2} \mathfrak{D}), f\left(x_{1}\right)\right)+\tilde{\psi}\left(f\left(x_{1}\right), f\left(x_{2}\right)\right)+\tilde{\psi}\left(f\left(x_{2}\right), \hat{f}(2 \mathfrak{l})\right) \\
& \leq \tilde{\psi}\left(f\left(x_{1}\right), f\left(x_{2}\right)\right)+\varepsilon \leq \sup _{a, b \in F} \tilde{\psi}(f(a), f(b))+\varepsilon
\end{aligned}
$$

Thus

$$
\psi \circ(\hat{f} \times \hat{f})(2 \mathfrak{W}, 2 \mathfrak{2}) \leq \inf _{F \in 2 \mathfrak{2} \cap 2 \pi} \sup _{a, b \in F} \tilde{\Psi} \circ(f \times f)(a, b)+\varepsilon .
$$

Since $\tilde{\psi} \circ(f \times f) \in \Gamma$ and by arbitrariness of $\varepsilon$, we conclude that $\psi \circ(\hat{f} \times \hat{f}) \in \hat{\Gamma}$.

Let cAUnif denote the full subcategory of AUnif consisting of all complete Hausdorff approach uniformities.

COROLLARY 3.7. cAUnif is a reflective subcategory of AUnif.

\section{Examples}

4.1. Extensions of pseudo-metrics. Any collection $\mathbb{D}$ of pseudo-metrics that is closed under the formation of finite suprema, is a basis for some approach uniformity $\Gamma$. In that case, we call $\mathfrak{D}$ a uniform gauge for $\Gamma$. We already noticed [Proposition 3.3] that if $\mathfrak{D}$ is a uniform gauge for $\Gamma$, then $\tilde{\mathbb{D}}:=\{\hat{d} \mid d \in \mathbb{D}\}$ is a basis for $\hat{\Gamma}$. As a matter of fact, $\tilde{\mathscr{D}}$ is a uniform gauge too. Moreover, $\tilde{\mathbb{L}}$ consists of the unique uniformly continuous extensions of the pseudo-metrics in $\mathfrak{D}$ from $X \times X$ to $\hat{X} \times \hat{X}$.

Proposition 4.1. Let $(X, \Gamma)$ be an approach uniformity and let $d \in \Gamma$ be a pseudometric. Then $\forall 2 \mathfrak{W}, 2 \mathfrak{\imath} \in \hat{X}$ :

$$
\hat{d}(2 \mathfrak{W}, 2 \mathfrak{l})=\sup _{M \in 2 \mathfrak{D}} \sup _{N \in \mathfrak{2} \mathfrak{l}} d(M, N) \text {. }
$$

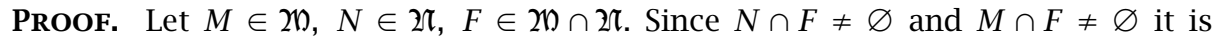
apparent that

$$
\forall \varepsilon>0, \exists p \in N, \exists q \in M: d(p, q) \leq \sup _{x, y \in F} d(x, y)+\varepsilon
$$


This is equivalent to the fact that

$$
\inf _{p \in N} \inf _{q \in M} d(p, q) \leq \sup _{x, y \in F} d(x, y)
$$

and therefore we have that

$$
\sup _{M \in 2 \mathfrak{Z}} \sup _{N \in 2 \mathfrak{2}} \inf _{p \in N} \inf _{q \in M} d(p, q) \leq \inf _{F \in 2 \mathfrak{2} \cap 2 \pi} \sup d(x, y) .
$$

Conversely, let $\varepsilon>0$ and choose $M \in \mathfrak{W}$ and $N \in \mathfrak{2}$ such that $\sup _{x, y \in M} d(x, y)<$ $\varepsilon / 2$ and $\sup _{x, y \in N} d(x, y)<\varepsilon / 2$. If $F:=M \cup N$, then $\forall x, y \in F, \forall p \in M, \forall q \in N$ : $d(x, y) \leq d(p, q)+\varepsilon$ since

- If $(x, y) \in N \times N$ or $(x, y) \in M \times M$ then this is trivial.

- If $x \in M$ and $y \in N$, then

$$
d(x, y) \leq d(x, p)+d(p, q)+d(q, y) \leq \frac{\varepsilon}{2}+d(p, q)+\frac{\varepsilon}{2} .
$$

Thus

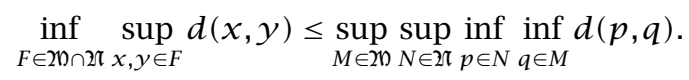

4.2. Metric and uniform approach uniformities. The categories pMET and Unif also allow a nice completion theory (cf. [8] for metric spaces, [10] for uniform spaces). As a matter of fact, the completion theory in AUnif generalizes these constructions.

Proposition 4.2. (a) Let $(X, \Gamma)$ be a metric approach uniform space, $\Gamma=\Gamma(d)$. Then $\hat{\Gamma}=\Gamma(\hat{d})$.

(b) Let $(X, \Gamma)$ be a uniform approach uniform space, $\Gamma=\Gamma(\mathfrak{U})$. Then $\hat{\Gamma}=\Gamma(\hat{\mathfrak{U}})$.

Proof. Part (a) is a special case of Proposition 4.1. Part (b) is a direct consequence of Proposition 3.2.

4.3. Initial structures. In this section, we show that, as in Unif, completion commutes with the formation of subspaces and products.

Proposition 4.3. Let $\left(f_{i}: X \rightarrow Y_{i}\right)_{i \in I}$ be an initial AUnif-source.

(1) The source of canonical extensions $\left(\hat{f}_{i}: \hat{X} \rightarrow \hat{Y}_{i}\right)_{i \in I}$ is initial too.

(2) If $j_{i}: Y_{i} \rightarrow \hat{Y}_{i}$ are the canonical injections and if

$$
e: X \rightarrow \prod_{i \in I} \hat{Y}_{i}: x \longmapsto\left(j_{i} \circ f_{i}(x)\right)_{i \in I},
$$

then $\hat{X} \cong \overline{e(X)}$.

Proof. The (categorical) proof (e.g., [9]) of the equivalent statements in Unif, can be imitated.

Proposition 4.4. If $Y$ is a subspace of $X$ in AUnif, then $\hat{Y}=\mathrm{cl}_{\hat{X}}(Y)$.

Proof. Apply Proposition 4.3(2) to the embedding $i: Y \rightarrow X$. 
Proposition 4.5. ${\widehat{\prod_{i \in I} Y}}_{i}=\prod_{i \in I} \hat{Y}_{i}$.

Proof. Since the source of projections $\left(\mathrm{pr}_{i}: \prod Y_{i} \rightarrow Y_{i}\right)_{i \in I}$ is initial, we have by Proposition 4.3(2), that (closures with respect to $\prod \hat{Y}_{i}$ )

$$
\widehat{\prod Y_{i}}=\overline{e\left(\prod Y_{i}\right)}=\overline{\prod Y_{i}}=\prod \hat{Y}_{i}
$$

4.4. Hyperspaces. Let $(X, d)$ be a metric space. In [6] it was shown that the hyperspace $\mathrm{CL}(X):=\{A \subset X \mid A$ closed $\}$ allows a natural approach uniformity generated by the $\infty p$-metrics $\left\{d_{H} \mid H \in 2^{(X)}\right\}$, where $\forall A, B \in \operatorname{CL}(X)$ :

$$
d_{H}(A, B):=\sup _{x \in H}|d(x, A)-d(x, B)| .
$$

Its uniform coreflection is the Wijsman uniformity (see [1]). We shall show that $\mathrm{CL}(\hat{X})=$ $\widehat{\mathrm{CL}(X)}$.

LEMMA 4.6. Let $X$ be a metric space. Then $\mathrm{CL}(X)$ is a dense subspace of $\mathrm{CL}(\hat{X})$ in AUnif.

Proof. In [7, Theorem 1.5], it is shown that $i: \mathrm{CL}(X) \rightarrow \mathrm{CL}(\hat{X}): A \mapsto \mathrm{cl}_{\hat{X}} A$ is a dense embedding in Unif. We only need to show that $\operatorname{CL}(X)$ carries the AUnif-subspace structure with respect to this embedding. Let $d$ denote the metric on $X$, and let $\hat{d}$ be the metric completion.

The initial approach uniformity for the singleton source $i$ is generated by $\infty p$ metrics of the form

$$
d_{K} \circ(i \times i)(A, B)=\sup _{x \in K}\left|\hat{d}\left(x, \mathrm{cl}_{\hat{X}}(A)\right)-\hat{d}\left(x, \mathrm{cl}_{\hat{X}}(B)\right)\right|,
$$

where $K$ is a finite subset of $\hat{X}$. If $K$ is a finite subset of $\hat{X}$ and $\varepsilon>0$, then there exists a finite subset $H \subset X$ such that $\forall x \in K, \exists y \in H: \hat{d}(x, y)<\varepsilon / 2$. Then $d_{K} \circ(i \times i) \leq$ $d_{H}+\varepsilon$. On the other hand, $\forall K \in 2^{(X)}: d_{K} \leq d_{i(K)} \circ(i \times i)$.

Proposition 4.7. Let $X$ be a metric space. Then $\operatorname{CL}(\hat{X})=\widehat{\operatorname{CL}(X)}$.

Proof. First notice that if $Y$ is complete, then its Wijsman uniformity (which is the uniform coreflection of $\mathrm{CL}(Y)$ ) is complete by [7, Theorem 1.5]. Hence $\mathrm{CL}(Y)$ is complete in AUnif. Since $\operatorname{CL}(X)$ is a dense subspace of $\operatorname{CL}(\hat{X})$ (Lemma 4.6), we obtain by Proposition 4.4 that $\widehat{\operatorname{CL}(X)}$ is the closure of $\operatorname{CL}(X)$ in $\widehat{\operatorname{CL}(\hat{X})}=\operatorname{CL}(\hat{X})$, i.e., $\widehat{\operatorname{CL}(X)}=$ $\mathrm{CL}(\hat{X})$.

4.5. Compactification. The Čech-Stone compactification of a topological space can be described in two essentially different ways: as a closed subspace of a product of the unit interval $[0,1]$ and as the underlying topology of the completion of the initial uniformity for all continuous functions into [0,1] (cf. [2]).

On the analogy of the former description a compactification in AP was defined in [4] (or [6]) as follows. (Suppose $[0,1]$ is equipped with the usual, i.e., euclidean, approach structure.) If $(X, \delta)$ is an approach space, then denote

$$
\mathfrak{K}^{\star}(X):=\{f: X \rightarrow[0,1] \mid f \text { is a contraction }\} .
$$


Suppose $X^{\prime}:=\prod_{f \in \mathfrak{K}^{\star}(X)}[0,1]$ is equipped with the product distance $\delta^{\prime}$. Consider $e:(X, \delta) \rightarrow\left(X^{\prime}, \delta^{\prime}\right): x \mapsto(f(x))_{f \in \mathfrak{K}^{\star}(X)}$ and let $\beta^{\star} X:=\overline{e(X)}$ be equipped with the subspace distance $\beta^{\star} \delta$. The space $\left(\beta^{\star} X, \beta^{\star} \delta\right)$ is called the compactification of $(X, \delta)$. This construction generalizes the Čech-Stone compactification in Top.

The alternative description mentioned above can be generalized in the context AUnif. (Henceforth, suppose $[0,1]$ is equipped with the usual, i.e., euclidean, approach uniformity.)

Proposition 4.8. Let $(X, \delta)$ be a Hausdorff uniform approach space. If $K^{\star}(X)$ is the initial approach uniformity for the source

$$
(f: X \longrightarrow[0,1])_{f \in \mathfrak{K}^{\star}(X)},
$$

and $\hat{\delta}$ is the underlying approach space of $\widehat{K^{\star}(X)}$, then $(\hat{X}, \hat{\delta})=\left(\beta^{\star} X, \beta^{\star} \delta\right)$.

Proof. By definition, the source

$$
\left(f:\left(X, K^{\star}(X)\right) \longrightarrow[0,1]\right)_{f \in \mathfrak{K}^{\star}(X)} .
$$

is initial. By Proposition 4.3(2) we obtain that $\left(\hat{X}, \widehat{K^{\star}(X)}\right) \cong(\overline{e(X)}, \Gamma)$ where $\Gamma$ is the relativization of product approach uniformity on $[0,1]^{\mathfrak{K}^{\star}(X)}$. Consequently (and using [6, Proposition 4.1]),

$$
(\hat{X}, \hat{\delta})=\left(\hat{X}, A\left(\widehat{K^{\star}(X)}\right)\right) \cong(\overline{e(X)}, A(\Gamma))=\left(\beta^{\star} X, \beta^{\star} \delta\right)
$$

Still on the analogy of the situation in Top and Unif, we have the following corollary.

Proposition 4.9. Let $(X, \delta)$ be a uniform approach space. Then $(X, \delta)$ is compact if and only if $\left(X, K^{\star}(X)\right)$ is complete.

4.6. Realcompactification. If in the above definition of compactification in AP [0,1] is replaced by $\mathbb{R}$ (equipped with the usual approach structure) and the stars are dropped (in particular $\mathfrak{K}(X):=\{f: X \rightarrow \mathbb{R} \mid f$ is a contraction $\}$ ), then the space $(\beta X, \beta \delta)$ is a generalization of the well-known Hewitt-Nachbin realcompactification in Top (cf. [2]).

Here too, we have an alternative description in terms of completion.

Proposition 4.10. let $(X, \delta)$ be a Hausdorff uniform approach space. If $K(X)$ is the initial approach uniformity for the source

$$
(f: X \rightarrow \mathbb{R})_{f \in \mathfrak{K}(X)},
$$

and $\hat{\delta}$ is the underlying approach space of $\widehat{K(X)}$, then $(\hat{X}, \hat{\delta})=(\beta X, \beta \delta)$.

COROLLARY 4.11. Let $(X, \delta)$ be a uniform approach space. Then $(X, \delta)$ is realcompact if and only if $(X, K(X))$ is complete. 
COROLLARY 4.12. Every realcompact uniform approach space is completely uniformizable. Every realcompact uniform approach space is AP-complete.

Proof. If $(X, \delta)$ is realcompact, then $K(X)$ is complete and induces $\delta$. By Proposition 2.3 we see that $(X, \delta)$ is AP-complete.

ACKNOWLEDGement. The second author is Aspirant of the Foundation for Scientific Research Flanders.

\section{REFERENCES}

[1] G. Beer and R. Lucchetti, Weak topologies for the closed subsets of a metrizable space, Trans. Amer. Math. Soc. 335 (1993), no. 2, 805-822. MR 93d:54023. Zbl 810.54011.

[2] L. Gillman and M. Jerison, Rings of Continuous Functions, D. Van Nostrand Co., Inc., Princeton, N.J.-Toronto-London-New York, 1960. MR 22\#6994. Zbl 093.30001.

[3] R. Lowen, Approach Spaces, The Clarendon Press Oxford University Press, New York, 1997. MR 98f:54002. Zbl 891.54001.

[4] R. Lowen and K. Robeys, Compactifications of products of metric spaces and their relations to $\hat{C}$ ech-Stone and Smirnov compactifications, Topology Appl. 55 (1994), no. 2, 163183. MR 94m:54063. Zbl 802.54018.

[5] R. Lowen and B. Windels, On the quantification of uniform properties, Comment. Math. Univ. Carolin. 38 (1997), no. 4, 749-759. MR 98m:54029.

[6] _ AUnif: a common supercategory of pMET and Unif, Internat. J. Math. Math. Sci. 21 (1998), no. 1, 1-18. MR 98j:54014. Zbl 890.54024.

[7] K. Morita, Completion of hyperspaces of compact subsets and topological completion of open-closed maps, General Topology and Appl. 4 (1974), 217-233. MR 50\#3175. Zbl 288.54009.

[8] C. G. C. Pitts, Introduction to Metric Spaces, Oliver \& Boyd [Longman Group Ltd.], Edinburgh, 1972. MR 57\#4112. Zbl 269.54019.

[9] G. Preuss, Theory of Topological Structures, D. Reidel Publishing Co., Dordrecht, 1988. MR 89m:54014. Zbl 649.54001.

[10] S. Willard, General Topology, Addison-Wesley Publishing Co., Reading, Mass.-London-Don Mills, Ont., 1970. MR 41\#9173. Zbl 205.26601.

LOWEN: UNIVERSITEIT ANTWERPEN, DEPARTEMENT WiSKUNDE-INFORMATICA, GROENENBORGERLAAN 171 B-2020, ANTWERPEN, BELGIUM

E-mail address: 1owen@ruca. ua.ac. be

Windels: DePARTMENT OF MATHEMATICS AND COMPUTER SCIENCE, UNIVERSiTy OF ANTWERP, RUCA, GROENENBORGERLAAN 171, 2020, ANTWERPEN, BELGIUM

E-mail address: winde1s@ruca.ua.ac.be 


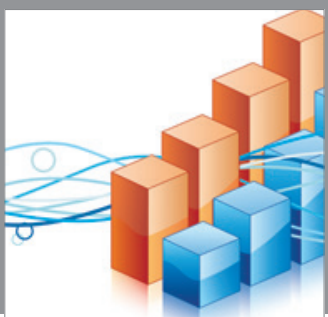

Advances in

Operations Research

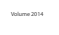

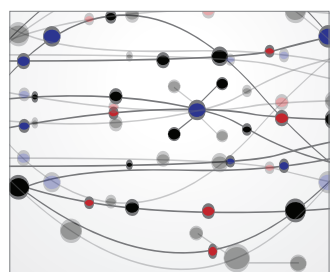

\section{The Scientific} World Journal
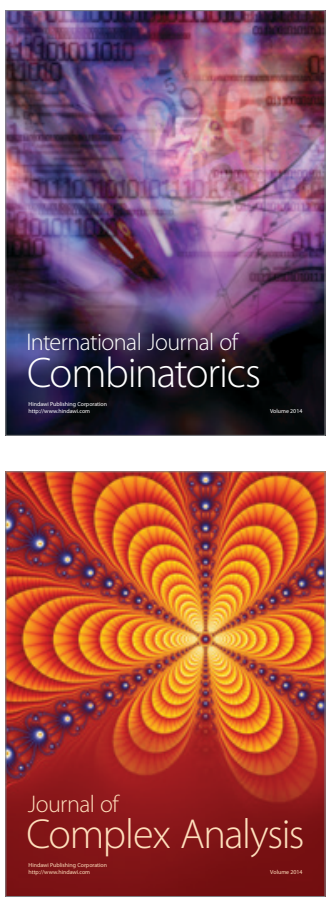

International Journal of

Mathematics and

Mathematical

Sciences
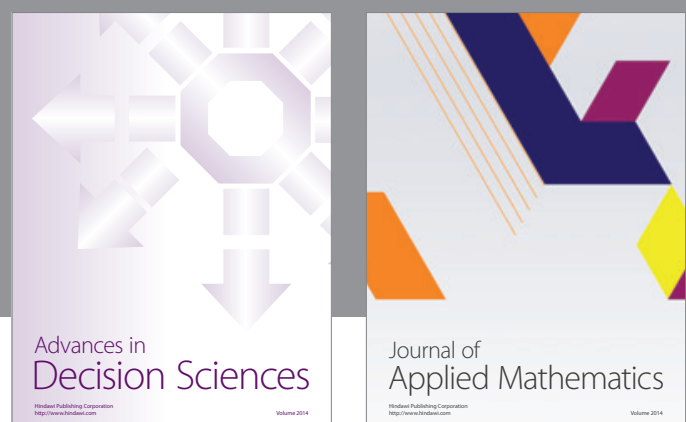

Journal of

Applied Mathematics
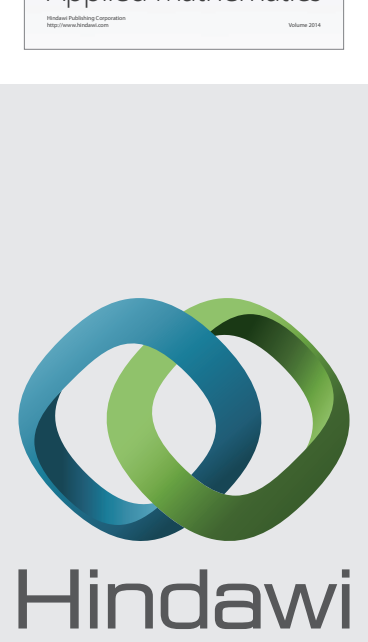

Submit your manuscripts at http://www.hindawi.com
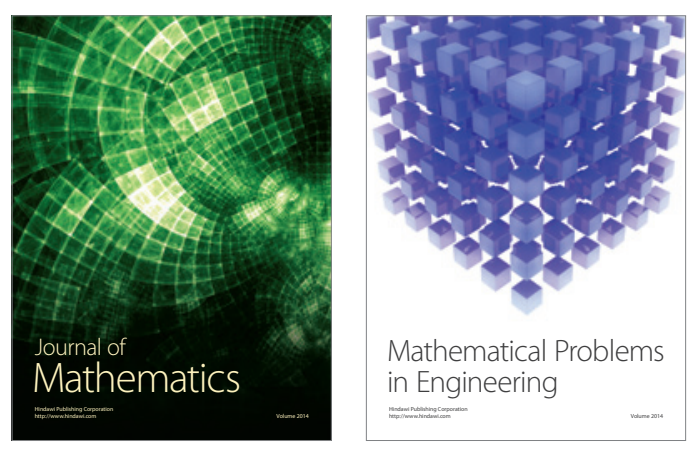

Mathematical Problems in Engineering
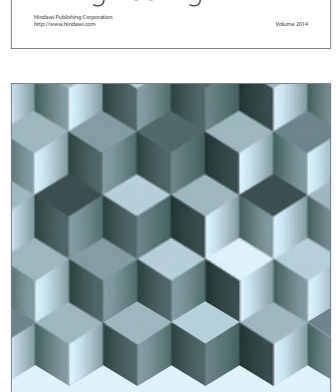

Journal of

Function Spaces
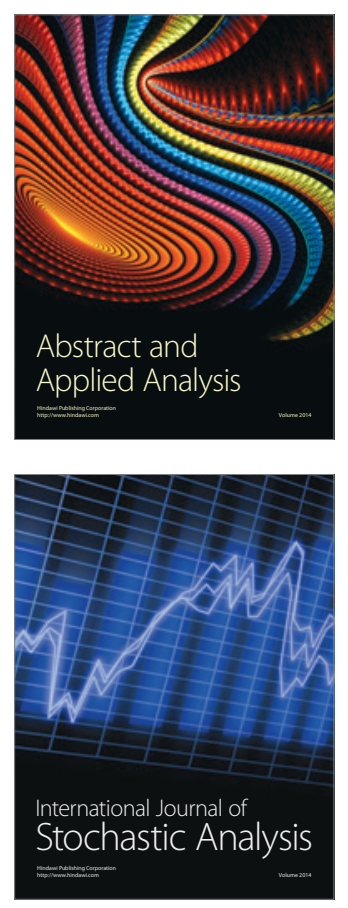

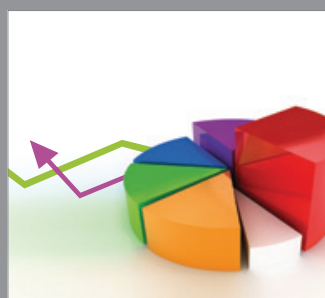

ournal of

Probability and Statistics

Promensencen
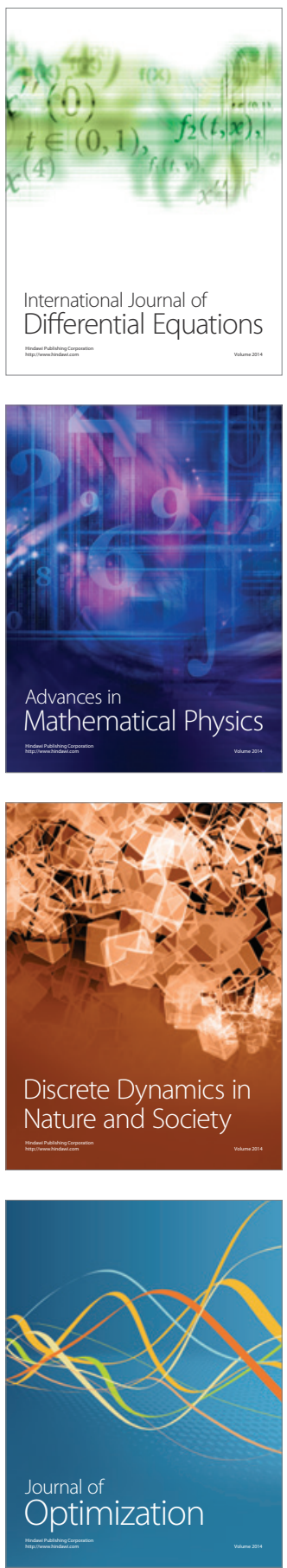